\title{
Automated analysis of three-dimensional stress echocardiography
}

\author{
K. Y. E. Leung • M. van Stralen • M. G. Danilouchkine • \\ G. van Burken • M. L. Geleijnse • J. H. C. Reiber • \\ N. de Jong • A. F. W. van der Steen • J. G. Bosch
}

Published online: 21 May 2011

(C) The Author(s) 2011. This article is published with open access at Springerlink.com

\begin{abstract}
Real-time three-dimensional (3D) ultrasound imaging has been proposed as an alternative for two-dimensional stress echocardiography for assessing myocardial dysfunction and underlying coronary artery disease. Analysis of 3D stress echocardiography is no simple task and requires considerable expertise. In this paper, we propose methods for automated analysis, which may provide a more objective and accurate diagnosis. Expert knowledge is incorporated via statistical modelling of patient data. Methods for identifying anatomical views, detecting endocardial borders, and classification of wall motion are described and shown to provide favourable results. We also present software developed especially for analysis of 3D stress echocardiography in clinical practice. Interobserver agreement in wall motion scoring is better using the dedicated software $(96 \%)$ than commercially available software not dedicated for this purpose $(79 \%)$. The developed tools may
\end{abstract}

K. Y. E. Leung $\cdot$ M. van Stralen • M. G. Danilouchkine •

G. van Burken $\cdot$ N. de Jong • A. F. W. van der Steen •

J. G. Bosch $(\triangle)$

Erasmus MC, Thoraxcenter - Biomedical Engineering,

Office Ee2302, P.O. Box 2040, 3000 CA, Rotterdam,

the Netherlands

e-mail: j.bosch@erasmusmc.nl

M. L. Geleijnse

Cardiology, Thoraxcenter, Erasmus MC,

Office Ba-304, P.O. Box 2040, 3000 CA, Rotterdam,

the Netherlands

J. H. C. Reiber

Division Image Processing, Radiology,

Leiden University Medical Center,

Leiden, the Netherlands

N. de Jong · A. F. W. van der Steen

ICIN - Interuniversity Cardiology Institute of the Netherlands,

Utrecht, the Netherlands provide useful quantitative and objective parameters to assist the clinical expert in the diagnosis of left ventricular function.

Keywords Three-dimensional imaging · Stress echocardiography. Statistical models

\section{Introduction}

Stress echocardiography (stress echo) is widely applied for evaluating cardiac function $[1,2]$. Motion of the left ventricle is assessed by comparing ultrasound images taken in rest and in stress stages. In the past decade, real-time three-dimensional (3D) imaging has become available for quantitative and objective assessment of cardiac function $[3,4]$. 3D imaging offers the opportunity to overcome the limitations of traditional two-dimensional stress echo [5]. With 3D imaging, non-foreshortened anatomical views can be defined since different view choices can be made after acquisition. Also, it provides better possibilities for quantifying the true 3D wall motion. However, due to suboptimal image quality, lower spatial and temporal resolution, and the sheer amount of data, quantitative analysis by hand is tedious and subjective.

Automated analysis methods may allow objective and faster assessment of clinical parameters such as left ventricular volume, ejection fraction and wall motion. In practice, the clinician makes a diagnosis using expert knowledge, gathered by analysing many patient images $[6,7]$. To develop successful automated methods which can deal with different pathologies and varying image quality, it makes sense to take expert knowledge into account.

In this paper, we present automated methods for 3D stress echo which make use of knowledge gained from databases of patient data. The knowledge is encompassed in so-called 


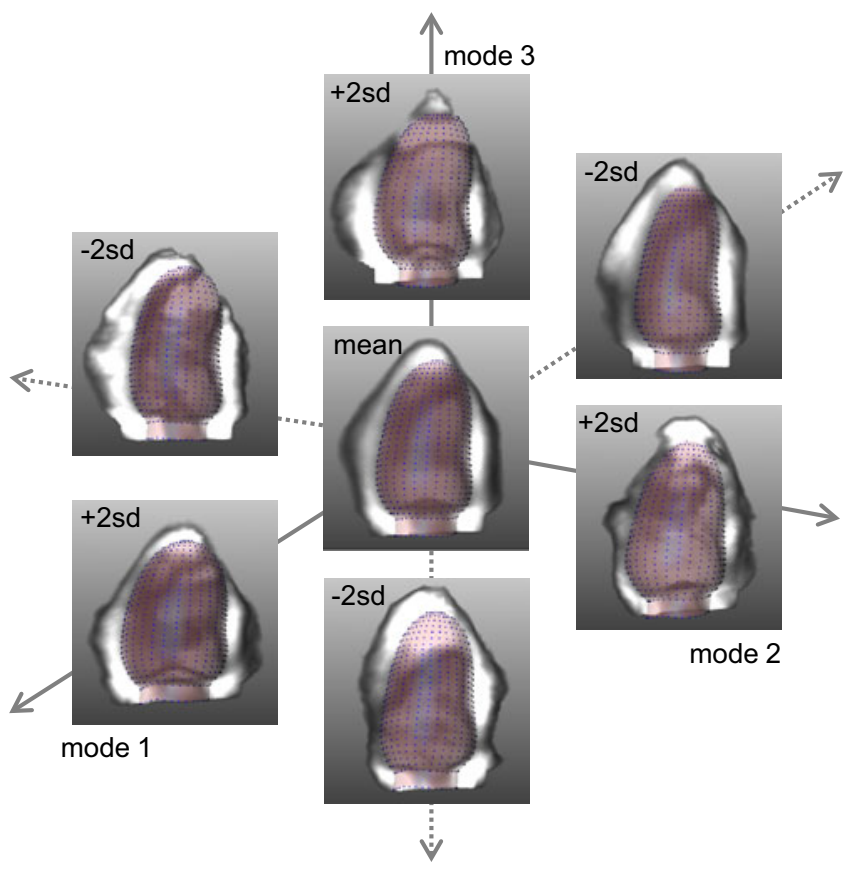

Fig. 1 Modes of variations of an appearance model, created by varying the model descriptors one at a time. The appearance model consists of a 'shape' (spatial coordinates) and a 'texture' (image intensity values) component. The average and the three most principal modes ( \pm 2 standard deviations) are shown

statistical models. Using Principal Component Analysis, patient variability can be represented concisely as mathematical descriptors that form a vector of orthogonal parameters [8]. The parameters to be modelled (for example, spatial coordinates of the endocardial border) are compared with the average parameters and typical variations in the patient database. Essentially, the methods are geared towards fitting the image data at hand to the model. In other words, the variation is estimated according to a set of extra criteria (such as image intensity values, 'smoothness' of the endocardial border, physically plausible motion, etc.).
Our proposed analysis scheme consists of three steps: identification of correct anatomical views in 3D data, detection of endocardial borders from which motion can be derived, and automated classification of motion. In addition, we present a software package which we have developed dedicated to analysis of 3D stress echo.

\section{Identification of anatomical views}

First we describe a method for detecting four-chamber, twochamber, and short-axis views in a 3D image. These views are modelled via appearance (image intensity values) and pose parameters (translation, rotation, and scaling). These parameters are identified by fitting the database model to the image at hand within an optimisation framework [9].

The views in a rest image can be used to extract the anatomically corresponding views in a stress image of the same patient. This is achieved via image registration [10], so that rest and stress images are aligned via minimisation of an objective criterion (in this case the correlation between image intensity values) [11]. Qualitative and quantitative evaluation in 20 end-diastolic and 20 end-systolic images shows that the views found are in many cases better (18\%) or similar (75\%) to the manual identification of views. Anatomically aligned views are thus obtained automatically, providing more possibilities for accurate visualisation and quantification.

\section{Quantification of volume and wall motion}

Detection of endocardial borders provides important clinical parameters such as volume, ejection fraction, and wall motion. Here, the active appearance model technique [8, 12] is adapted to detect borders in an end-diastolic 3D echocardiogram. The endocardial borders are modelled via spatial coordinates, distributed in a cylindrical and spherical

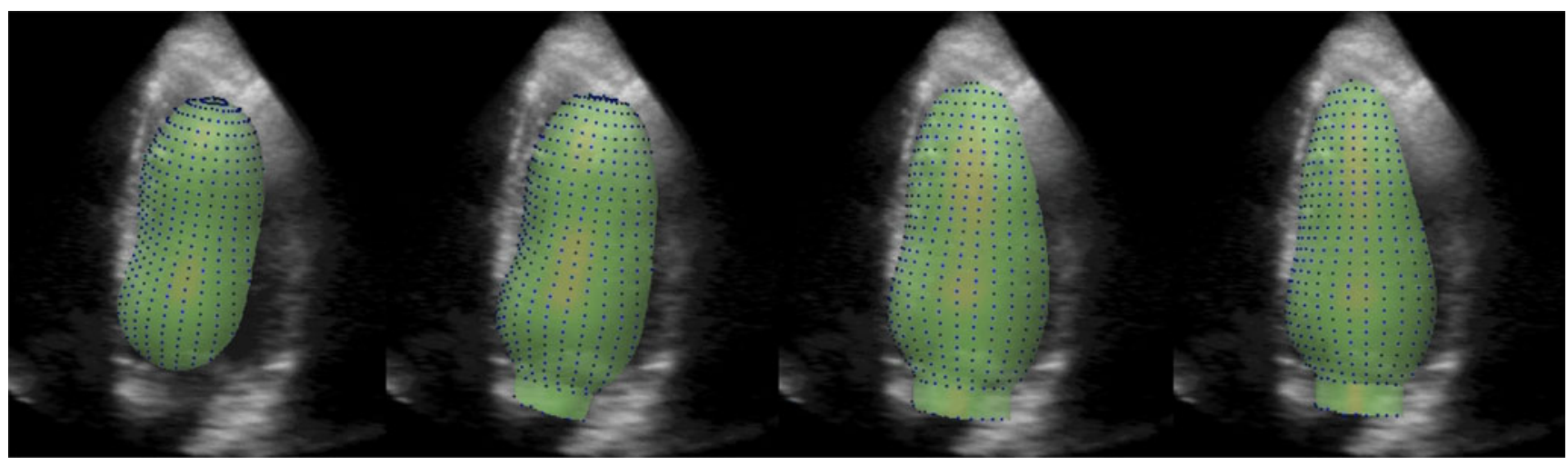

start

result

Fig. 2 Endocardial border detection in a 3D image. The borders are described as spatial coordinates (blue dots) in 3D. The initial position of the borders and the results after 10,20, and 39 (final) results are shown 




Fig. 3 Modes of variation as calculated by Principal Component Analysis (PCA), which depict global variations in endocardial border motion, versus the local modes of variation after Orthomax rotation. The amount of the variation is colour-coded. The local modes are more concise and suitable for automatic classification of wall motion abnormalities

representation which is suited to the shape of the left ventricle (Fig. 1). Image intensity values are mapped to a normal (Gaussian) distribution for appropriate statistical analysis [12]. The model represents variations of ventricular shapes and the typical appearance of the ventricle and myocardium in echocardiograms, including the typical artifacts. The model is trained for image analysis by estimating the relation between model parameters and image change via regression analysis: in the actual matching, the intensity difference between model and image is used to update the model's parameters and drive the model closer to the image (Fig. 2). Evaluation on 99 patient images shows a successful matching in $91 \%$ of cases, with a median surface error of $2.65 \mathrm{~mm}$ (average $2.91 \mathrm{~mm}$, standard deviation $1.03 \mathrm{~mm}$ )[13].

The borders throughout the cardiac cycle are obtained by tracking the motion of the cardiac wall. A 3D optical flow algorithm is applied, which uses spatial and temporal intensity gradients to track the endocardial border between two time frames. To ensure an overall temporal continuity, the tracking method takes into account physically plausible motion, learned statistically from a patient database. Typical image artifacts, such as echo drop-out and near-field artifacts, are detected and dealt with appropriately using an expectation maximisation based classification approach [14]. The method is capable of tracking the endocardial borders accurately (in

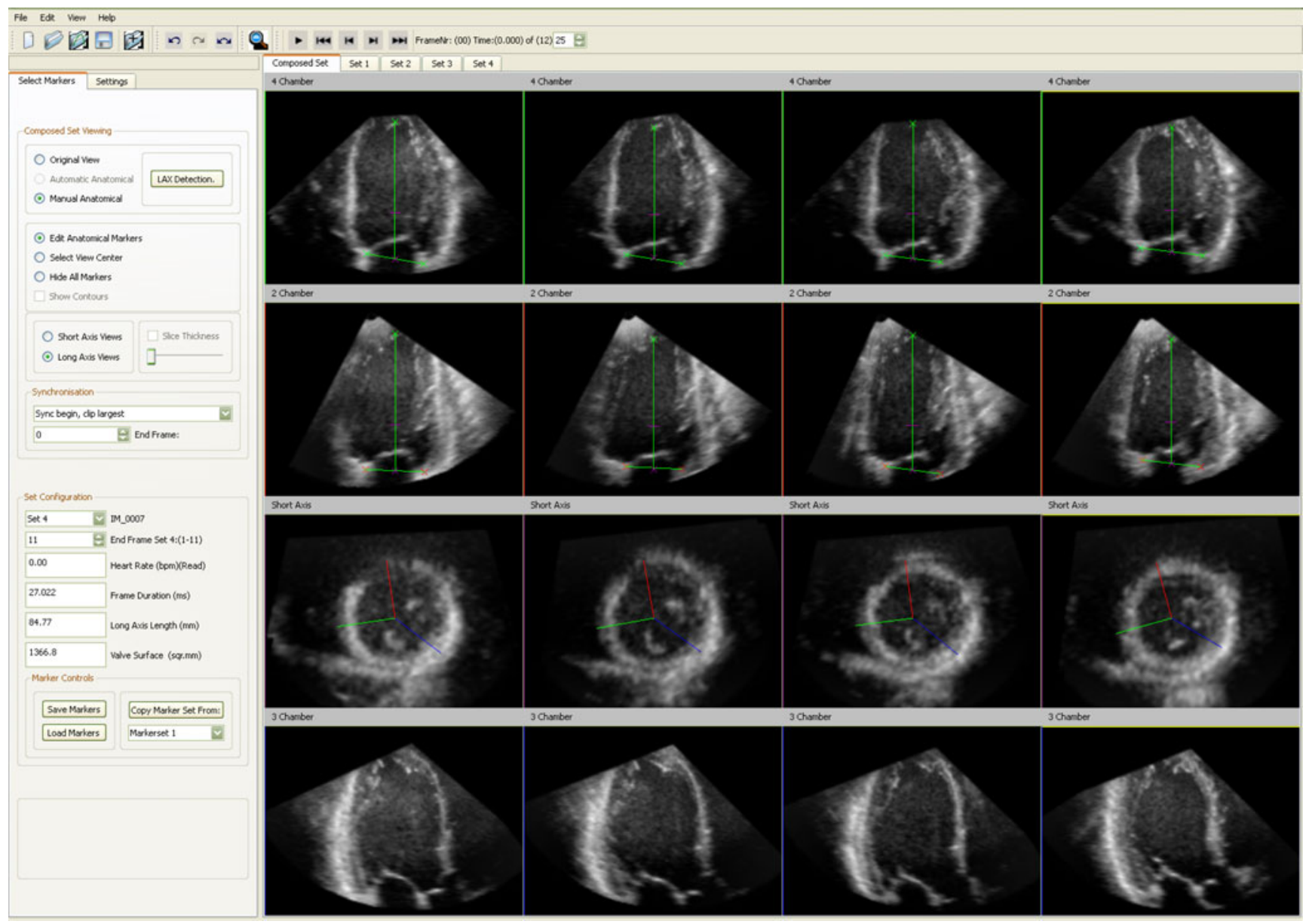

Fig. 4 Software dedicated to 3D stress analysis, allowing side-by-side viewing of images acquired at rest and in varying stages of stress. Anatomical four-chamber, two-chamber, short-axis, and long-axis views are shown 
35 patients: surface errors: $1.2 \pm 0.5 \mathrm{~mm}$, volume errors: $1.4 \pm$ $6.7 \mathrm{ml}$, ejection fraction errors: $0.9 \pm 4.8 \%$ ).

\section{Automated motion classification}

Statistical models of the endocardial border motion throughout the cardiac cycle can be used to discriminate between normal and pathological motion patterns. The statistical model is transformed using the Orthomax rotation criterion, so that the local wall motion can be represented using fewer descriptors (Fig. 3) [6]. An automated classification method is then applied to the descriptors. Wall motion classification is demonstrated in 129 two-dimensional echocardiographic sequences. Using these models, local motion abnormalities can be detected accurately in $77-91 \%$ of the cases. In principle, the method can be applied directly to 3D borders; the evaluation is a subject of further investigation.

\section{Tools for clinical practice}

To enable 3D stress echocardiography in routine clinical practice, we have also developed a software package dedicated to 3D stress echo [15]. The essential functionality for analysing 3D stress echo is investigated: side-by-side, synchronised display of anatomically aligned rest and stress images (Fig. 4). Two expert observers analysed 34 noncontrast clinically available rest and stress images visually. Interobserver agreement for segmental myocardial ischaemia is better using the dedicated software (96\%) than commercially available software $(79 \%)$ without these functionalities.

\section{Conclusion}

In this paper, we have presented automated methods for identification of anatomical views, detection of endocardial borders, and classification of wall motion abnormalities. The proposed methods make use of statistical modelling of expert knowledge from patient databases. Considerable advances have been made to allow a more objective and accurate analysis. In particular, these methods are better capable of handling typical image artifacts and following expert tracing conventions. We have also demonstrated the advantages of using dedicated software for the analysis of 3D stress echo in the clinical setting. Based on this research, opportunities remain for further development, e.g. in the analysis of contrast echocardiograms, technical enhancement of image quality and spatiotemporal resolution, and improvement of clinical workflow. The developed tools can provide useful quantitative and objective parameters to help the clinical expert in the diagnosis of left ventricular function.
Acknowledgments This research is supported by the Dutch Technology Foundation STW (grant 06666), applied science division of NWO and the Technology Program of the Ministry of Economic Affairs, the Netherlands

Open Access This article is distributed under the terms of the Creative Commons Attribution Noncommercial License which permits any noncommercial use, distribution, and reproduction in any medium, provided the original author(s) and source are credited.

\section{References}

1. Geleijnse ML, Fioretti PM, Roelandt JR. Methodology, feasibility, safety and diagnostic accuracy of dobutamine stress echocardiography. J Am Coll Cardiol. 1997;30(3):595-606.

2. Hoffmann R, Marwick TH, Poldermans D, et al. Refinements in stress echocardiographic techniques improve inter-institutional agreement in interpretation of dobutamine stress echocardiograms. Eur Heart J. 2002;23(10):821-9.

3. Matsumura Y, Hozumi T, Arai K, et al. Non-invasive assessment of myocardial ischaemia using new real-time three-dimensional dobutamine stress echocardiography: comparison with conventional twodimensional methods. Eur Heart J. 2005;26(16):1625-32.

4. Soliman OI, Krenning BJ, Geleijnse ML, et al. Quantification of left ventricular volumes and function in patients with cardiomyopathies by real-time three-dimensional echocardiography: a head-to-head comparison between two different semiautomated endocardial border detection algorithms. J Am Soc Echocardiogr. 2007;20(9):1042-9.

5. Jenkins C, Chan J, Hanekom L, Marwick T. Accuracy and feasibility of online 3-dimensional echocardiography for measurement of left ventricular parameters. J Am Soc Echocardiogr. 2006;19(9):1119-28.

6. Leung KYE, Bosch JG. Segmental wall motion classification in echocardiograms using compact shape descriptors. Acad Radiol. 2008;15(11):1416-24.

7. Geleijnse ML, Krenning BJ, Nemes A, et al. Factors affecting sensitivity and specificity of dobutamine stress echocardiography. J Am Soc Echocardiogr. 2009;22:1199-208.

8. Cootes TF, Edwards GJ, Taylor CJ. Active appearance models. IEEE Trans Pattern Anal Mach Intell. 2001;23(6):681-5.

9. Leung KYE, van Stralen M, van Burken G, et al. Sparse appearance model based registration and segmentation of 3D echocardiographic images. Proc IEEE Ultrason Symp. 2006:2413-6.

10. Mäkelä T, Clarysse P, Sipilä O, et al. A review of cardiac image registration methods. IEEE Trans Med Imaging. 2002;21 (9):1011-21.

11. Leung KYE, Van Stralen M, Nemes A, et al. Sparse registration for three-dimensional stress echocardiography. IEEE Trans Med Imaging. 2008;27(11):1568-79.

12. Bosch JG, Mitchell SC, Lelieveldt BPF, et al. Automatic segmentation of echocardiographic sequences by active appearance motion models. IEEE Trans Med Imaging. 2002;21(11):1374-83.

13. Leung KYE, van Stralen M, van Burken G, de Jong N, Bosch JG. Automatic active appearance model segmentation of $3 \mathrm{D}$ echocardiograms. Proc Int Symp Biomed Imaging. 2010;2010:320-3.

14. Leung KYE, Danilouchkine MG, van Stralen M, de Jong N, van der Steen AFW, Bosch JG. Probabilistic framework for improving tracking in artifact-prone 3D echocardiograms. Med Image Analysis. 2010;14:750-8.

15. Nemes A, Leung KYE, van Burken G, et al. Side-by-side viewing of anatomically aligned left ventricular segments in threedimensional stress echocardiography. Echocardiography. 2009;26 (2):189-95. 